\title{
High heat flux test results for a thermal break DEMO divertor target and subsequent design and manufacture development
}

\author{
Adomas Lukenskas $^{1 *}$, T. R. Barrett ${ }^{1}$, M. Fursdon ${ }^{1}$, F. Domptail ${ }^{1}$, F. Schoofs ${ }^{1}$, H. Greuner ${ }^{2}$, G. Dose ${ }^{3}$, \\ S. Roccella ${ }^{4}$, E. Visca ${ }^{4}$, F. Gallay ${ }^{5}$, M. Richou ${ }^{5}$, J.-H. You ${ }^{2}$ \\ ${ }^{1}$ CCFE, Culham Science Centre, Abingdon, Oxon, OX14 3DB, UK \\ ${ }^{2}$ Max Planck Institute for Plasma Physics, Boltzmann Str. 2, 85748 Garching, Germany \\ ${ }^{3}$ Università di Roma “Tor Vergata”, Dipartimento di Ingegneria Industriale, Via del Politecnico 1, 00133 Rome, Italy \\ ${ }^{4}$ ENEA Frascati, Fusion \& Technology for Nuclear Safety, via E. Fermi 45, 00044 Frascati, Italy \\ ${ }^{5}$ CEA, IRFM, F-13108 Saint Paul Lez Durance, France
}

\begin{abstract}
This paper focuses on the development of the water-cooled divertor target concept known as Thermal Break, which was carried out in two phases. In Phase 1, six small scale mock-ups were fabricated and subjected to high heat flux (HHF) testing of up to $25 \mathrm{MW} / \mathrm{m}^{2}$ and thermal cycling of up to 500 cycles at $20 \mathrm{MW} / \mathrm{m}^{2}$. All six mock-ups survived the campaign and maintained $20 \mathrm{MW} / \mathrm{m}^{2}$ heat exhaust capability. Detailed examination of mock-ups was carried out to understand the damage mechanisms. One mock-up, which was tested beyond its design intent at 500 cycles, shows signs of progressive damage. Potential damage modes were identified and influenced subsequent Phase 2 mock-up design. Although there are signs of tungsten surface cracking, the predominant damage mode is not by "deep cracking" but substantial permanent deformation in the interlayer features.

Therefore, in Phase 2 the manufacturing procedure was updated, the interlayer grooves were given stress-relieving radii which have significantly reduced the interlayer plastic strain range. Interlayer design parameters were selected following the use of response surface-based design search and optimization. Mock-ups of the Phase 2 design have been manufactured and HHF testing is planned within 2018.
\end{abstract}

\section{Keywords: CuCrZr, Divertor Target, Thermal Break, High Heat Flux, Brazing}

\section{Introduction}

The design of the DEMO divertor is a critical research topic within nuclear fusion field. Conditions for divertor plasma facing components (PFCs) such as low tritium retention, high sputtering resistance, radiation damage resistance, and ability to sustain high heat flux (HHF) load of 10 's $\mathrm{MW} / \mathrm{m}^{2}$ significantly complicate design, material selection and manufacturing processes. Within EUROfusion [1] different design, evaluation and fabrication approaches are being considered. Concepts such as a liquid metal divertor are also considered within Europe [2].

This work considers the Thermal Break concept [3], which is an evolution of the ITER tungsten/CuCrZr monoblock design [4] in which the copper interlayer has machined features to reduce conductivity and stiffness which alleviates stress in the PFC. This work is a continuation of that previously presented by M. Fursdon et al. [5] in which development and testing of the Phase 1 thermal break divertor target design delivering $20 \mathrm{MW} / \mathrm{m}^{2}$ heat load capability was presented. This article presents the lessons learned from designing, fabricating and testing these $1^{\text {st }}$ phase thermal break mock-ups, together with the latest design and fabrication changes for the $2^{\text {nd }}$ phase thermal break DEMO divertor mock-ups.

\section{Phase 1 post HHF test results}

The $1^{\text {st }}$ phase thermal break mock-ups were evaluated for their performance under HHF using the IPP test facility GLADIS [6]. The mock-ups were subjected to 20 $\mathrm{MW} / \mathrm{m}^{2}$ (nominal power density of an approximately
Gaussian distribution) for progressively $100,150,300$ cycles and achieved their design intent. One of the mockups (mock-up no. 6) was further tested to enable damage mode analysis. It was subjected to 500 cycles (cycles of $10 \mathrm{~s}$ ) at $20 \mathrm{MW} / \mathrm{m}^{2}$. Initially the mock-up maintained uniform temperature distribution across all blocks. After 500 cycles the mock-up continued to maintain heat exhaust capability, however some tungsten blocks exhibited higher temperature than others (see Fig. 1).

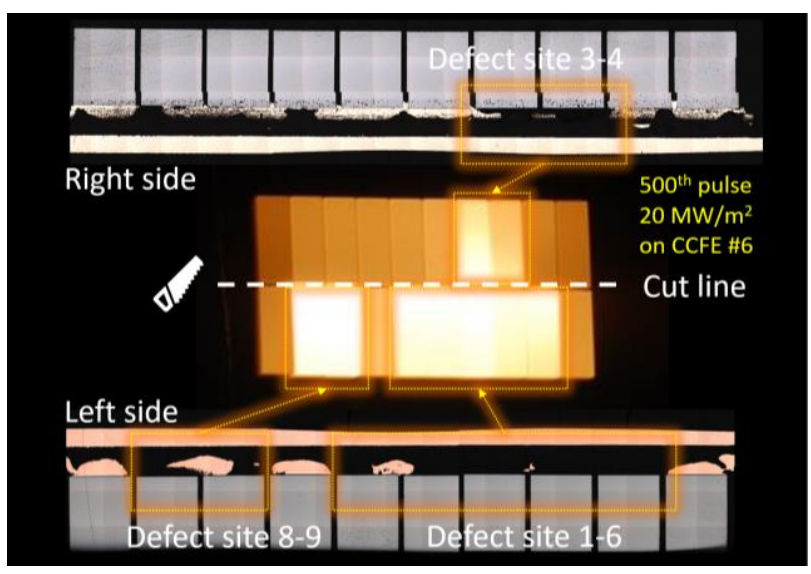

Fig. 1. CCD camera image of mock-up \#6 under 20 $\mathrm{MW} / \mathrm{m}^{2}$ at GLADIS (IPP, Germany). Displayed on the top and bottom of the image are microscope images of the axial cross-section. Defect sites 3-4 on the right side, and defect sites 1-6, 8-9 on the left side match the observation of overheated blocks during the mock-up test. 


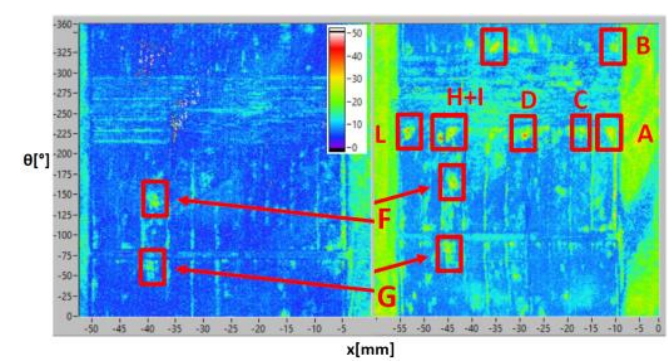

Fig. 2. Ultrasonic images (C-scans) at 2nd brazing interface (between $\mathrm{Cu}$ interlayer and $\mathrm{W}$ ) of CCFE mock-up \#6 captured at ENEA Frascati (Italy), before (left) and after (right) HHF testing. The horizontal axis reports the axial displacement of the probe inside the pipe $(\mathrm{z}[\mathrm{mm}])$, while the vertical axis gives the rotation angle (theta [degrees]) The pixel colour gives the amplitude of the considered variable inside of a chosen depth range (maximum amplitude of the pressure signal) Red squares highlight the most pronounced defects detected at this interface. The scale indicates the defect depth.

After HHF testing, the mock-up was inspected using non-destructive ultrasonic imaging at ENEA Frascati (Italy) [7], (see for example Fig. 2). The ultrasonic inspection showed an increased number of defect sites in the $\mathrm{W} / \mathrm{Cu}$ interface, as well as accentuation of the initial fabrication defects (Fig. 2, G and F). This corresponded with subsequent observations when the mock-up was sectioned axially and inspected with an optical microscope (Fig. 3a). Microscopy revealed cracks and voids in the braze joint already highlighted by the Ultrasonic Testing (UT) before the HHF test (defect G in Fig. 2 is evident in Fig. 3 a). Ultrasonic inspection, also, detects defects inside the interlayer as the degradation of spokes (see [8]). Other mock-ups subjected to high cycle HHF testing were cut radially. Nearly all mock-ups showed some degree of degradation in the interlayer (see example in Fig. 3 b). This progressive permanent deformation in the interlayer is the most common damage mode throughout the Phase 1 mock-ups tested.

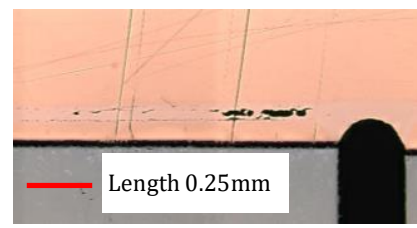

a. Defects in the braze joint at the bottom of mock-up No 6. (Defect G in Fig 2.)

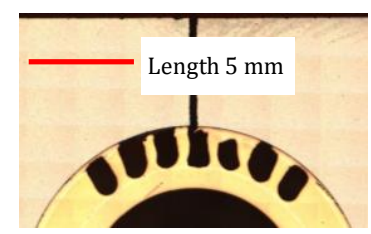

b. Degradation of spokes in the interlayer, mockup No 5. sharp corners are no longer present
Fig. 3. Defects in the interlayer.

\subsection{Phase 1 post HHF discussion}

The detailed inspection of the mock-ups after the HHF testing gave valuable insights on potential routes to improve the thermal break design for the $2^{\text {nd }}$ phase. Permanent deformation in the interlayer features was identified as the predominant damage mode. A formal Finite Element (FE) model optimization procedure is therefore needed to explore different interlayer topologies and refine the design to reduce this interlayer cyclic plastic deformation. Furthermore, since it was observed that initial fabrication defects are accentuated during HHF cycling, Phase 2 mock-ups would need to be manufactured with fewer defects. Therefore, the Phase 2 design would use a single braze layer, instead of two as in Phase 1 , to minimize potential fabrication defects and reduce the potential of failure by delamination and crack propagation in the braze joints.

\section{Phase $\mathbf{2}$ design and fabrication development}

The Phase 2 monoblock design is larger in dimensions than Phase 1 in order to unify major dimensions with other designs being assessed across EUROfusion WPDIV [1]. These fixed design constraints are shown in figure 4 .

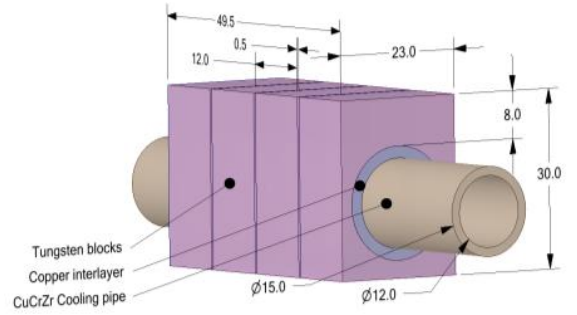

Fig. 4. Summary of geometric constraints in $\mathrm{mm}$ for $2^{\text {nd }}$ phase mock-ups (agreed within EUROfusion WPDIV). The design optimization took these dimensions as fixed.

Using an FE analysis and formal optimization procedure an updated thermal break design was produced (Fig. 5, Phase-2). The new Phase 2 design has obround interlayer features, whereas in the previous design the interlayer features had sharp corners. This was incorporated to prevent premature fatigue, buckling and delamination of interlayer spokes. The new design also has a single braze joint to reduce fabrication complexity and potential defects which could be accentuated during HHF cycling.

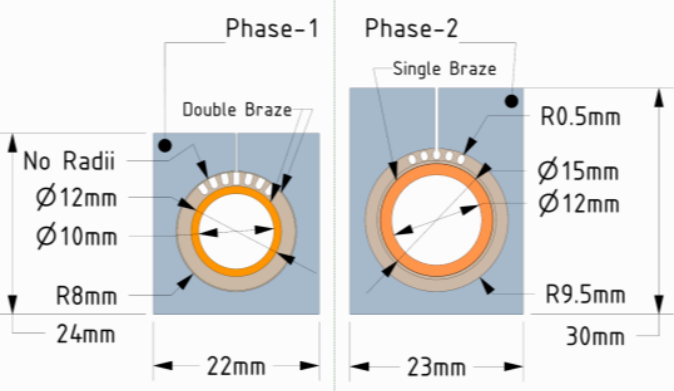

Fig. 5. Phase 1 monoblock compared to Phase 2 monoblock design.

\subsection{Design optimization by Finite Element Analysis and Design of Experiments}

A finite element analysis (FEA) model was built in ANSYS Workbench Release 16.2 [9] which was used for objective function calculations. The model was called according to Design of Experiments (DoE), and for a 
range of different design criteria and design variables a response surface (interpolating cubic spline) is calculated and searched for improved designs or simply plotted to enable examination of the design space. Key variables are the number of obround holes in the interlayer and interlayer thickness (see Fig. 6). The criteria and objectives, as well as materials properties, model's thermal loads, and solution steps were based on Fursdon's draft Plastic Analysis Procedure (PAP) [10]. Eleven performance metrics were considered in total and the main parameters are given in Table 1.

Table 1. Key design performance parameters for heat flux of $20 \mathrm{MW} / \mathrm{m}^{2}$.

\begin{tabular}{ll}
\hline Parameter & $\begin{array}{l}\text { Objective/ } \\
\text { Constraint }\end{array}$ \\
\hline $\begin{array}{l}\text { Von Mises fluctuation standby to Q in } \\
\text { pipe (for 3Sm) }\end{array}$ & $<2.7 \times 10^{8} \mathrm{~Pa}$ \\
Peak interlayer temperature & $<885^{\circ} \mathrm{C}$ \\
Peak CuCrZr pipe temperature & $<450^{\circ} \mathrm{C}$ \\
$\begin{array}{l}\text { Total Strain in interlayer fluctuation } \\
\text { standby to Q (for fatigue) }\end{array}$ & Minimize \\
The peak first principal stress in the & $<4.33 \times 10^{8} \mathrm{~Pa}$ \\
\hline
\end{tabular}

Since DoE requires many runs of the FEA model, a simplified model type was developed and validated. The simplified model represents a quarter of a monoblock with two symmetry planes. Validation of this simplified model was achieved by comparing the results with the full mockup simulation. The discrepancy between results of the full and the simplified model is within $10 \%$. The simplified model allowed each optimization to be conducted in a manageable time frame.

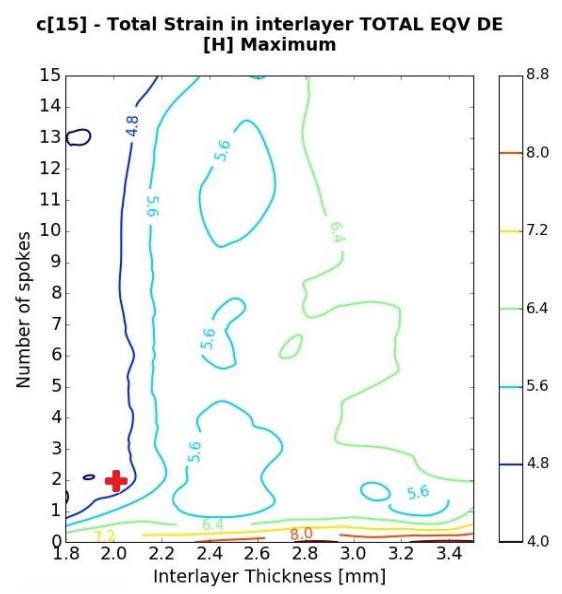

Fig. 6. Example of a single response surface for a given parameter - total strain in the interlayer. Red cross indicates Phase 2 design selection.

Sixteen different design topologies were considered, and thousands of FEA model simulations were solved. A python script was written to automatically generate and plot response surface charts from the ANSYS results.

The study indicated that the design achieving a good compromise between competing objectives of low interlayer strain range, low pipe stress, and low temperatures, would use a centrally split monoblock design with $2 \mathrm{~mm}$ partially continuous interlayer, $1 \mathrm{~mm}$ wide obround holes spaced $11^{\circ}$ apart. This selected Phase 2 design is shown in Fig. 5. The response surface for interlayer strain range, showing the selected design point, given in Fig. 6.

\subsection{Updated Phase 2 Fabrication Process}

The selected design was produced by machining tungsten (W) to size and casting oxygen-free high conductivity (OFHC) copper into the bore of the block. The OFHC copper was left $1 \mathrm{~mm}$ proud of $\mathrm{W}$ block to allow subsequent thermal break features to be machined into the interlayer. The blocks together with casting were provided by ALMT (Japan). Thermal break features were wire eroded in the interlayer. Parts were precisely bored to match the outer diameter $15.00 \pm 0.01 \mathrm{~mm} \mathrm{CuCrZr}$ alloy pipe. The pipes were machined from a solid block of $\mathrm{CuCrZr}(0.5-1.2 \% \mathrm{Cr}, 0.02-0.07 \% \mathrm{Zr}$, rest $\mathrm{Cu})$. Before assembly, the machined $\mathrm{CuCrZr}$ pipes were subjected to full brazing/heat treatment cycle and the resulting surface oxide and impurity layer was mechanically removed. All parts were cleaned and assembled along with braze alloy foil $(50 \% \mathrm{Cu} / 50 \% \mathrm{Au})$. The pipe and monoblock assemblies were positioned in bespoke braze tooling which supports the parts and ensures alignment during the brazing procedure.

Brazing was carried out in a vacuum furnace following a single braze/hardening procedure (Fig. 7). In order to achieve good wetting properties, assemblies were heated to $1020^{\circ} \mathrm{C}$ at $1.8 \times 10^{-5}$ mbar vacuum. The parts were cooled to $950^{\circ} \mathrm{C}$ to solidify the braze before a nitrogen gas quench. The quench was carried out by purging nitrogen gas to prevent formation of precipitates and to keep the $\mathrm{CuCrZr}$ alloy in solution annealed state. After the quench, the parts were vacuum aged at $480^{\circ} \mathrm{C}$ for 2 hours for to achieve the required hardness.

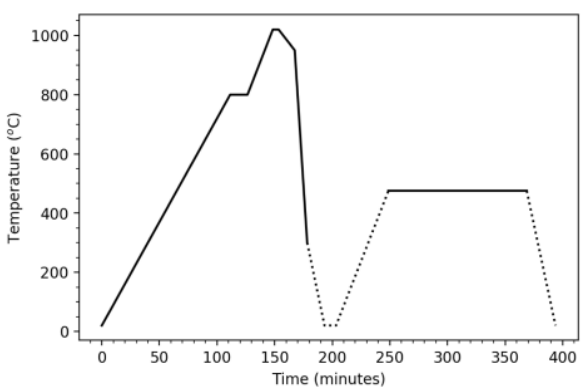

Fig. 7. A single brazing and heat treatment procedure used for fabrication of Phase 2 mock-ups.

\section{Results and discussion}

In total four, $2^{\text {nd }}$ phase mock-ups were produced (Fig. 8). Optical microscopy inspection of witness samples, both in radial and axial directions, showed no defects in the braze joint. Ultrasonic imaging results from ENEA Frascati (Italy) supports the initial observations since no fabrication defects were found in the mock-ups. Preliminary SATIR tests [11] showed no thermal imperfections. SEM, as well as X-ray fluorescence 
imaging used during fabrication development and mockup inspection phase showed no contamination by other materials during brazing and handling of mock-ups. Results from neutron scattering spectroscopy [12] showed a precipitate size distribution within the pipe to be nearly unaffected by thermal mass of the monoblocks on the pipe. This was confirmed by Vickers hardness test results in different axial locations of the pipe showing that hardness of $\mathrm{CuCrZr}$ pipe is even throughout the entire length, with typical values ranging from 108 to 116 .

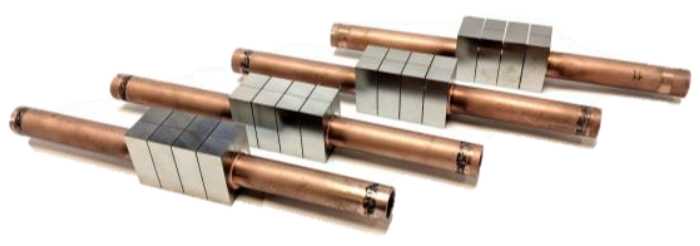

Fig. 8. Completed Phase 2 mock-ups.

Despite the more challenging geometric constraints of Phase 2, the calculated design performs better on most criteria than the Phase 1 design. The comparison of models from both phases suggests a reduction by $28 \%$ in the interlayer strain - the parameter leading to the dominant damage mode in the Phase 1 tests.

The FEA model was adjusted prior to HHF load simulations to consider the residual manufacturing stress state and geometry changes caused by different thermal expansion during brazing procedure. The new geometry and stress state were verified by comparing the topology deformation results from Phase 2 FEA model to the asmanufactured final mock-up, as shown in Fig. 9. The FEA model match the shape of the as-manufactured final mock-up extremely well. This comparison gives confidence that the FEA model is reporting accurately the thermally induced strain, and also that the actual mock-up was produced as intended.

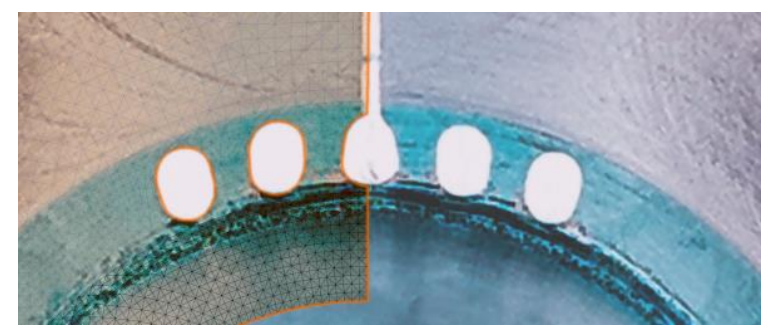

Fig. 9. Photograph of a Phase 2 mock-up after fabrication, overlaid with the true scale deformation results calculated by FEA model (in orange).

\section{Conclusions}

HHF testing of Phase 1 mock-ups proved that thermal break concept could potentially be implemented in the divertor design. However, it has been shown that HHF cycling can eventually accentuate initial fabrication defects and degrade the braze joint, causing formation of axial cracks and voids. Also, damage mode analysis of Phase 1 mock-ups indicated that progressive permanent deformation in the interlayer features is the most common damage mode.
A method to accurately simulate the behaviour of the full mock-up on a simplified model was developed and validated. It was shown that DoE optimization method can be used to significantly improve important mock-up design parameters. Using $\mathrm{FE}$ analysis and formal optimization procedure a refined Phase 2 thermal break design was produced.

Compared to phase-1 fabrication procedure described in [5] an improved fabrication procedure was developed, and it was shown that a single braze joint can be successfully manufactured and yield a defect free joint. Four mock-ups of Phase 2 design were fabricated and further NDT and HHF testing is planned within 2018.

\section{Acknowledgments}

This work has been carried out within the framework of the EUROfusion Consortium and has received funding from the Euratom research and training programme 20142018 under grant agreement No 633053 and from the RCUK Energy Programme [grant number EP/P012450/1] The views and opinions expressed herein do not necessarily reflect those of the European Commission.

\section{References}

[1] J.-H. You, et al., European DEMO divertor target: Operational requirements and material-design interface, Nucl. Mater. Energy 9 (2016) 171-176.

[2] J. H. You, et al., Conceptual design studies for the European DEMO divertor: Rationale and first results, Fus. Eng. And Des., 109-111, 2016, 1598-1603.

[3] T. R. Barrett, Enhancing the DEMO divertor target by interlayer engineering, Fus. Eng. And Des., 98-99, 2015, 1216-1220.

[4] T. Hirai et al., Use of tungsten material for the ITER divertor, Nucl. Mater. Energy 9 (2016) 616-622.

[5] M. Fursdon et al., The development and testing of the thermal break divertor monoblock target design delivering $20 \mathrm{MW} / \mathrm{m}^{-2}$ heat load capability, Phys. Scr. T170 (2017) 014042.

[6] H. Greuner, B. Böswirth, T. R. Barrett, F. Crescenzi, F. Gallay, K. Hunger, M. Richou, E. Visca, A.v. Müller, J.H. You, Progress in high heat flux testing of European DEMO divertor mock-ups, Fus. Eng. Des. (2019).

[7] S. Roccella et al., Non-destructive methods for the defect detection in the ITER high heat flux components, Fus. Eng. Des. 86 (2011) 1791-6.

[8] G. Dose et al., Ultrasonic analysis of tungsten monoblock divertor mock-ups after high heat flux test, SOFT 2018.

[9] ANSYS Academic [Computer Software]. Workbench Release 16.2, Canonsburg, Pennsylvania: ANSYS, 2016.

[10] M. Fursdon, Plastic Analysis Procedure for Assessing Divertor Target Design (PAP), Draft - 2016.

[11] R. Guigon et al., Innovative image processing techniques applied to the thermographic inspection of PFC with SATIR facility, Fus. Eng. Des. 84 (2009) 859-863.

[12] F. Schoofs et al. Microstructure and mechanical property mapping of $\mathrm{CuCrZr}$ with complex and non-uniform thermal history 2018, SOFT 2018. 